\title{
Neuroprotective effect of acute melatonin treatment on hippocampal neurons against irradiation by inhibition of caspase-3
}

\author{
JIANGUO LI ${ }^{1-3}$, GUOWEI ZHANG ${ }^{3}$, ZHUANGZHI MENG $^{3}$, LINGZHAN WANG $^{3}$, \\ HAIYING LIU ${ }^{3}$, QIANG LIU ${ }^{2}$ and BATU BUREN ${ }^{1}$ \\ ${ }^{1}$ Laboratory of Biomedicine, Department of Hemopathic Tumor of Mongolian Medicine, \\ The Affiliated Hospital of Inner Mongolia University for The Nationalities, Neimenggu Tongliao, Inner Mongolia 028007; \\ ${ }^{2}$ Tianjin Key Laboratory of Molecular Nuclear Medicine, Institute of Radiation Medicine, \\ Chinese Academy of Medical Science and Peking Union Medical College, Tianjin 300192; \\ ${ }^{3}$ Department of Human Anatomy, The School of Medicine of Inner Mongolia University for The Nationalities, \\ Neimenggu Tongliao, Inner Mongolia 028007, P.R. China
}

Received January 4, 2015; Accepted February 23, 2016

DOI: $10.3892 /$ etm.2016.3215

\begin{abstract}
Neuronal cell apoptosis is associated with various factors that induce neurological damage, including radiation exposure. When administered prior to exposure to radiation, a protective agent may prevent cellular and molecular injury. The present study aimed to investigate whether melatonin exerts a neuroprotective effect by inhibiting the caspase cell death pathway. Male Sprague-Dawley rats were administered melatonin (100 mg/kg body weight) $30 \mathrm{~min}$ prior to radiation exposure in red light during the evening. In order to elucidate whether melatonin has a neuroprotective role, immunohistochemistry, terminal deoxynucleotidyl transferase dUTP nick-end labeling, Nissl staining, reverse transcription-quantitative polymerase chain reaction, reactive oxygen species analysis and western blotting were performed. At $24 \mathrm{~h}$ post-melatonin treatment, caspase-3 mRNA and protein expression levels were significantly decreased. These results demonstrated that melatonin may protect hippocampal neurons via the inhibition of caspase- 3 when exposed to
\end{abstract}

Correspondence to: Dr Batu Buren, Laboratory of Biomedicine, Department of Hemopathic Tumor of Mongolian Medicine, The Affiliated Hospital of Inner Mongolia University for The Nationalities, 1742 Huolinhe Street, Neimenggu Tongliao, Inner Mongolia 028007, P.R. China

E-mail: burenbatutlmd@126.com

Dr Qiang Liu, Tianjin Key Laboratory of Molecular Nuclear Medicine, Institute of Radiation Medicine, Chinese Academy of Medical Sciences and Peking Union Medical College, 238 Baidi Road, Nankai, Tianjin 300192, P.R. China

E-mail: liuqiang70@126.com

Key words: melatonin, caspase-3, reactive oxygen species, hippocampus irradiation. Therefore, caspase-3 inhibition serves a neuroprotective and antioxidant role in the interventional treatment of melatonin. The results of the present study suggested that melatonin may have a potential therapeutic effect against irradiation; however, further studies are required in order to elucidate the underlying antioxidant mechanisms.

\section{Introduction}

Neuronal cell apoptosis is associated with various factors that induce neurological damage, including radiation exposure (1). Free radicals produced by the interaction between ionizing radiation and the biological system attack the components of cells, resulting in cellular damage and apoptosis $(2,3)$. To date, two apoptotic pathways have been extensively characterized, one of which is triggered by the engagement of cell surface death receptors with their specific ligands, and the other is a mitochondrial pathway, triggered by alterations in internal cellular integrity induced by numerous stimuli $(4,5)$. Subsequently, these two pathways induce the activation of caspases (6), which hydrolyze important structural and functional proteins of the cell, ultimately leading to apoptosis (7). Caspases are synthesized in the cell as inactive zymogens and require activation to be functional (7). Radiation induces caspase activation through the mitochondrial pathway, which includes the mitochondrial integration of apoptotic signals and the subsequent release of cytochrome $c$, Smac and apoptosis-inducing factor into the cytosol (8). This release facilitates the assembly of the apoptosome, which activates caspase- 9 and, in turn, leads to the activation of caspases-3, -6 and -7 (9).

Caspase-mediated cell death is associated with the pathogenesis of neuronal degeneration along with other factors such as oxidative damage and inflammation. In addition to activating cell death, previous studies have demonstrated that caspase- 3 also has fundamental roles in signal transduction $(10,11)$. Reactive oxygen species (ROS) are byproducts 
of the normal cellular metabolism of oxygen; however, a dramatic increase in ROS levels may lead to oxidative stress. It has been demonstrated that ROS induced by ionizing radiation are capable of triggering oxidative cellular damage and stimulating the activation of intracellular signaling pathways (12). The brain, which is a major metabolizer of oxygen with relatively poor protective antioxidant mechanisms, is particularly vulnerable to the ROS. In recent years, research in this field has focused on antioxidant agents that are suitable as radiation countermeasures (13-15). An effective protective agent against irradiation administered prior to exposure to radiation may protect cells from cellular and molecular injury (16).

Melatonin ( $N$-acetyl-5-methoxytryptamine; Mel), which is a neurohormone and the major product of the pineal gland, may be a novel therapeutic agent for the treatment of various disorders associated with inflammation and oxidative stress (17). It has been reported that Mel was able to improve short and long-term neurobehavioral deficits and attenuate hippocampal impairments following hypoxia in neonatal mice (18). In addition to neutralizing ROS species, Mel also acts via the stimulation of various anti-oxidative systems and stabilizes cell membranes (19). As such, Mel modulates the gene expression levels of numerous protective enzymes to reduce apoptosis and lipid peroxidation $(20,21)$. Mel has previously been demonstrated to improve the survival rates of mice when administered prior to irradiation exposure (22). The hippocampus is a region of active proliferation and neurogenesis within the brain. It has previously been demonstrated that ionizing radiation induces the apoptosis of neural cells within the subventricular zone of the lateral ventricles and the subgranular zone of the hippocampus in the adult brain (23). Furthermore, previous studies have demonstrated that the caspase-dependent cytotoxicity of ionizing radiation in hippocampal neurons is induced by oxidative stress $(24,25)$.

The present study aimed to investigate whether Mel inhibits the caspase cell death pathway to protect hippocampal neurons from irradiation-induced apoptosis. Furthermore, the underlying mechanisms of this phenomenon within cells were studied in order to elucidate whether Mel may be a novel therapeutic agent for the prophylactic treatment of irradiation.

\section{Materials and methods}

Animals and reagents. A total of 18 male Sprague-Dawley rats, weighing 200-220 g and aged 6-8 weeks, were maintained in individual cages for 1 week at $22 \pm 2^{\circ} \mathrm{C}, 40-60 \%$ humidity and under a 12-h light-dark cycle, with ad libitum access to food and water. All animal experiments were conducted in accordance with a protocol approved by the Institutional Animal Care and Use Committee of the Institute of Radiation Medicine, Chinese Academy of Medical Sciences (Tianjin, China). Animals were randomly assigned into three groups ( $n=6 /$ group): Irradiation (IR) group, irradiation with Mel (IR + Mel) group and control (Con) group. Mel was purchased from ImmunoWay Biotechnology Company (Newark, DE, USA).

Mel administration. Rats in the IR + Mel group were administered Mel (100 mg/kg body weight) by intraperitoneal injection; the IR and Con groups were treated with an equal volume of isotonic $\mathrm{NaCl}$ solution (Fuyu Fine Chemical Co., Ltd., Tianjin, China) as a vehicle, with and without the proceeding irradiation, respectively. All treatments were performed 30 min prior to radiation exposure in red light at 6 p.m.

Irradiation. Rats were placed in ventilated plexiglass containers $(30 \times 25 \times 30 \mathrm{~cm}$; Nanfang Organic Glass Factory, Tianchang, China) and administered total body irradiation (TBI) using 137 Cs $\gamma$ rays (Cammacell-40; Atomic Energy, Mississauga, ON, Canada) at a dosage of $1.0 \mathrm{~Gy} / \mathrm{min}$ (26). Rats in the IR and IR + Mel groups received a total of 4.0 Gy TBI. Rats in the control group were placed in identical containers for the same period without irradiation.

Tissue preparation. At $24 \mathrm{~h}$ post-experimental intervention, the rats were sacrificed by an overdose with intraperitoneally administered sodium pentobarbital (50 mg/kg; Beijing Biosynthesis Biotechnology Co., Ltd., Beijing, China) and immediately treated with a cardiac perfusion of $4 \%$ paraformaldehyde (CellChip Biotechnology Co., Ltd., Beijing, China). The hippocampi were harvested and cut into $12-\mu \mathrm{m}$ coronal sections (3 rats/group) using a CM 3000 cryostat (Leica Microsystems GmbH, Wetzlar, Germany) and were subsequently placed on glass slides and stored at $-80^{\circ} \mathrm{C}(27)$.

Immunohistology, terminal deoxynucleotidyl transferase dUTP nick end-labeling (TUNEL) and cresyl violet $(C V)$ staining. A standard immunohistochemical analysis was conducted according to a previous study (28). Briefly, coronal sections were air dried for $15 \mathrm{~min}$, post-fixed in $10 \%$ formalin (Hangzhou Norming Biological Technology Co., Ltd., Hangzhou, China) for $15 \mathrm{~min}$, washed twice in phosphate-buffered saline and then processed for immunostaining with rabbit anti-active caspase-3 polyclonal antibody (1:1,000; cat. no. ab2302; Abcam, Cambridge, MA, USA). This was followed by incubation with horseradish peroxidase-conjugated goat anti-rabbit $\operatorname{IgG}$ (1:3,000; cat. no. ta140003; OriGene Technologies, Inc., Beijing, China) and then 3,3'-diaminobenzidine tetrahydrochloride (Sigma-Aldrich, St. Louis, MO, USA). Subsequently, the sections were visualized under a light microscope (LSM-510; Carl Zeiss AG, Oberkochen, Germany).

DNA fragmentation was detected using a TUNEL kit (In Situ Cell Death Detection Kit, POD; Roche Diagnostics, Indianapolis, IN, USA) according to the manufacturer's protocol and as described previously (29). Briefly, sections were incubated for $90 \mathrm{~min}$ at $37^{\circ} \mathrm{C}$ with TUNEL reaction mixture. Positive control sections were incubated with $200 \mathrm{U} / \mathrm{ml}$ DNase I (Gibco; Thermo Fisher Scientific, Inc., Waltham, MA, USA) for $5 \mathrm{~min}$ prior to fixation. Negative control sections underwent the same procedure but terminal deoxynucleotidyl transferase was omitted from the reaction buffer to evaluate nonspecific labeling. TUNEL cell counts were performed on brain sections $(n=3)$ from the hippocampi. TUNEL-positive cells were averaged from the counts of three adjacent brain sections of a rat. The sections were visualized using the Eclipse Ti-U inverted microscope (Nikon Corporation, Tokyo, Japan) with an excitation/emission wavelength of 500/550 nm (green).

$\mathrm{CV}$ staining was performed in order to detect the Nissl body in the neuronal cytoplasm and to identify the basic 

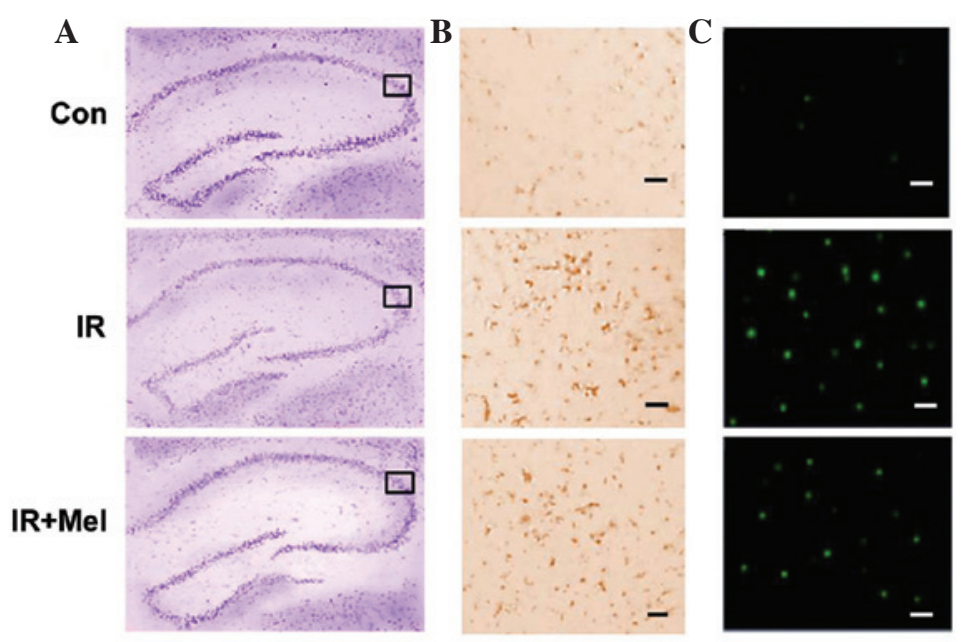

D

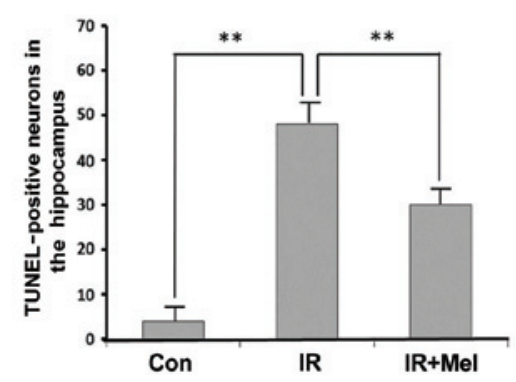

Figure 1. Representative images of photomicrographs stained with (A) cresyl violet, (B) caspase-3 and (C) TUNEL in the CA3 region of hippocampi. (D) TUNEL-positive neurons in hippocampi. The box indicates the image positioning of immunohistology and TUNEL. Scale bars, $50 \mu \mathrm{m} .{ }^{* *} \mathrm{P}<0.01 \mathrm{vs}$. the IR group. TUNEL, terminal deoxynucleotidyl transferase dUTP nick-end labeling; con, control; IR, irradiation; Mel, melatonin.
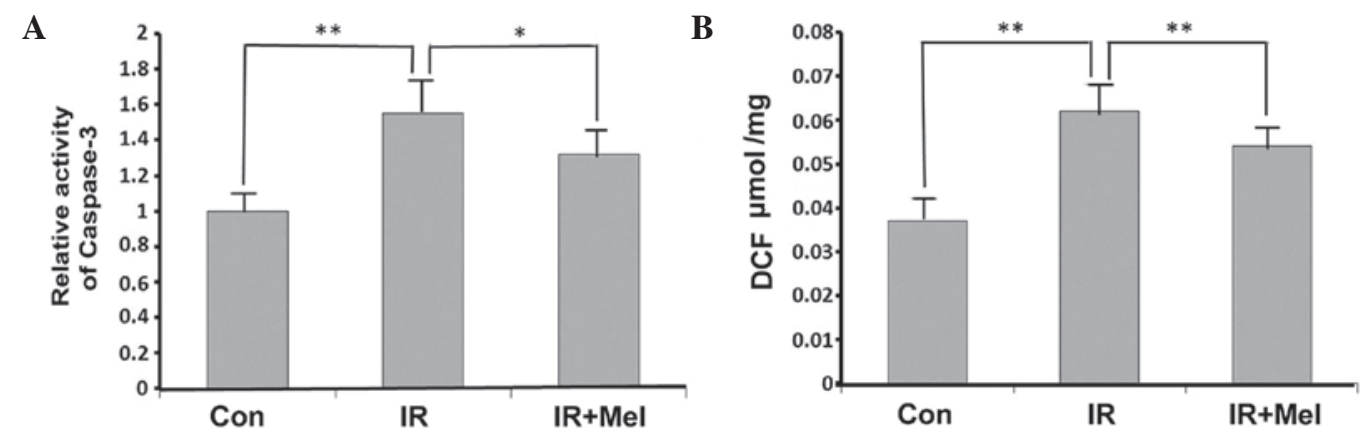

Figure 2. (A) Caspase-3 activity assay, expressed as fold change relative to the Con group. (B) DCF fluorescence intensity. ${ }^{*} \mathrm{P}<0.05$ and ${ }^{* *} \mathrm{P}<0.01$ vs. the IR group. Data are presented as the mean \pm standard deviation. Con, control; IR, irradiation; Mel, melatonin; DCF, dichlorofluorescein probe.

neuronal structure of necrotic neurons in the brain and spinal cord. Sections were rinsed in tap and distilled water, and subsequently stained in $0.1 \%$ CV solution (CellChip Biotechnology Co., Ltd.) for 3-10 min. Following rinsing in distilled water, the sections were differentiated in 95\% ethyl alcohol (Sangon Biotech Co., Ltd., Shanghai, China), dehydrated in 100\% alcohol and cleared with xylene (Sangon Biotech Co., Ltd.), prior to mounting with permanent mounting medium (Yantuo Biological Technology Co., Ltd., Shanghai, China). The Nissl body was stained purple-blue (28).

Western blot analysis. Western blotting was performed according to a standard procedure as described previously (29). Briefly, the rats were sacrificed at $24 \mathrm{~h}$ following irradiation, and the hippocampi ( $\mathrm{n}=3 /$ group) were obtained. Total protein was isolated from the hippocampi using radioimmunoprecipitation assay buffer (Beyotime Institute of Biotechnology, Jiangsu, China), according to the manufacturer's protocol. The homogenates were centrifuged at $21,890 \mathrm{x} \mathrm{g}$ for $30 \mathrm{~min}$ at $4^{\circ} \mathrm{C}$ and the protein concentration in the supernatant was determined spectrophotometrically by measuring the absorbance at $595 \mathrm{~nm}$ (A595 nm). Equal volumes $(20 \mu \mathrm{g})$ of protein were mixed with loading buffer containing 5\% 2-mercaptoethanol (Hangzhou Norming Biological Technology Co.,
Ltd.), heated for $5 \mathrm{~min}$ at $95^{\circ} \mathrm{C}$ and separated by $10 \%$ sodium dodecyl sulfate-polyacrylamide gel electrophoresis, followed by immunoblotting onto polyvinylidene difluoride membranes (EMD Millipore, Billerica, MA, USA). After blocking with $5 \%$ milk in Tris-buffered saline supplemented with $0.1 \%$ Tween-20, the membranes were incubated overnight at $4^{\circ} \mathrm{C}$ with rabbit anti-cleaved caspase- 3 polyclonal antibody (1:1,000; cat. no. ab2302; Abcam) and rabbit anti- $\beta$-actin monoclonal antibody (1:500; cat. no. 4967L; Cell Signaling Technology, Inc., Danvers, MA, USA, followed by incubation with HRP-conjugated goat anti-rabbit IgG (1:1,000; cat. no. ta140003; OriGene Technologies, Inc.) for $2 \mathrm{~h}$ at room temperature. Proteins were detected using enhanced chemiluminescence (ECL reagents; GE Healthcare Life Sciences, Little Chalfont, UK) and exposed to radiographic film (Hyperfilm ECL; GE Healthcare Life Sciences) .The relative amount of protein was normalized to $\beta$-actin and analyzed using the Gel-Pro Analyzer software, version 4.0 (Media Cybernetics, Inc., Rockville, MD, USA).

Reverse transcription-quantitative polymerase chain reaction $(R T-q P C R)$. Tissue samples were initially homogenized and total RNA was extracted from the tissues using TRIzol reagent (Invitrogen; Thermo Fisher Scientific, Inc.) according 
A

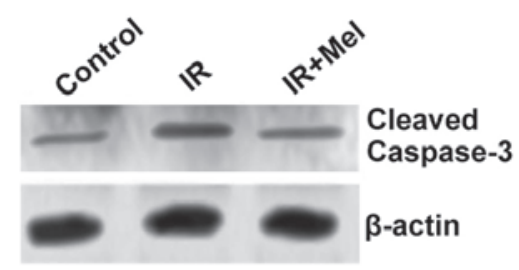

B

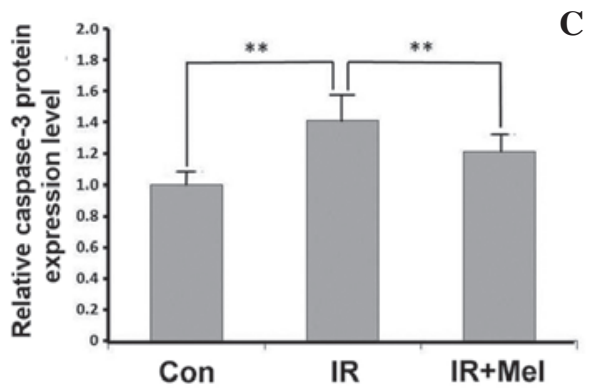

C

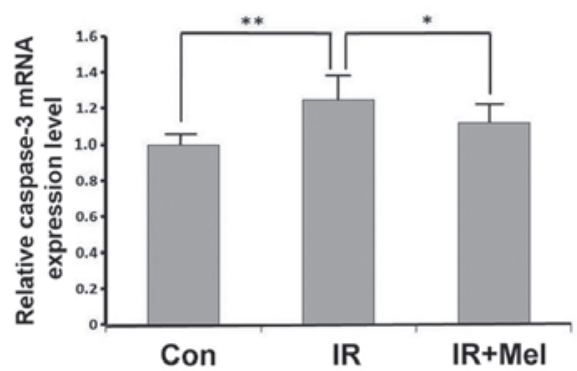

Figure 3. Effects of Mel on the expression level of caspase-3. (A) Western blot analysis, (B) protein expression levels and (C) mRNA expression levels of caspase-3, expressed as the mean \pm standard deviation relative to caspase- 3 levels in the control group. ${ }^{*} \mathrm{P}<0.05$ and ${ }^{* *} \mathrm{P}<0.01$ vs. the IR group. Con, control; IR, irradiation; Mel, melatonin.

to the manufacturer's protocol. RNA was treated with DNase (TURBO DNA-free ${ }^{\mathrm{TM}}$ kit; Thermo Fisher Scientific, Inc.) and $3 \mu \mathrm{g}$ RNA was used for cDNA synthesis, as previously described (29). Briefly, RNA was reverse transcribed into cDNA using a using an iScript ${ }^{\mathrm{TM}}$ Select cDNA Synthesis kit (Bio-Rad Laboratories, Inc., Hercules, CA, USA). PCR was performed using the 2X PCR Master Mix (Beyotime Institute of Biotechnology), an ABI PRISM ${ }^{\circledR} 7500$ Sequence Detection system (Applied Biosystems; Thermo Fisher Scientific, Inc.) and the following primers: Caspase-3, forward 5'-AATTCA AGGGACGGGTCATG-3' and reverse 5'-GCTTGTGCG CGTACAGTTTC-3'; and GAPDH, forward 5'-ATGACA TCAAGAAGGTGGTG-3' and reverse 5'-CATACCAGG AAATGAGCTTG-3' (Sangon Biotech Co., Ltd.). The PCR cycling conditions were as follows: $95^{\circ} \mathrm{C}$ for $20 \mathrm{sec}$, followed by 50 cycles at $95^{\circ} \mathrm{C}$ for $3 \mathrm{sec}$ and $60^{\circ} \mathrm{C}$ for $30 \mathrm{sec}$, and then a final extension step at $72^{\circ} \mathrm{C}$ for $5 \mathrm{~min}$. The cycle threshold $(\mathrm{Cq})$ values of caspase- 3 were normalized to the $\mathrm{Cq}$ values of the GAPDH housekeeping gene using the $2^{-\Delta \Delta \mathrm{Cq}}$ method (30) All samples were analyzed in triplicate A negative control and an RT-minus control were used to verify the results of the first strand cDNA synthesis step.

ROS analysis and caspase-3 activation assay. A 2',7'-dichlorodihydrofluorescein diacetate (DCF) fluorogenic probe (Hangzhou Boda Biological Technology Co., Ltd, Hangzhou, China) was used to assess the production of ROS, as previously described (31). The activities of caspase-3 were analyzed using a fluorogenic caspase assay with Ac-DEVD-AFC (BD Pharmingen, San Diego, CA, USA) as the substrate. The results were expressed as the fold change, compared with the control, according to technique described by Li et al (28).

Statistical analysis. Statistical analysis was performed using SPSS 13.0 software (SPSS, Inc., Chicago, IL, USA). Statistical comparison of the results was performed using paired Student's t-test. $\mathrm{P}<0.05$ was considered to indicate a statistically significant difference.

\section{Results}

Mel administration attenuates irradiation-induced neuronal damage. The Nissl body was successfully stained purple-blue (Fig. 1A). Microscopic examination of the hippocampal sections stained with $\mathrm{CV}$ showed pathological changes in the IR and IR \pm Mel groups, as compared with the control group (Fig. 1A). Viable neurons had a deeply stained cytoplasm and lightly stained nucleus. Immunohistochemical labeling of caspase-3 was more intense in the hippocampi of the IR group than in the IR + Mel group; whereas staining was more intense in the IR + Mel group than the control group in the cytoplasm (Fig. 1B). TUNEL-positive cells were not clearly observed in the CA3 region of the control group, whilst TUNEL-positive staining was detected in the nuclear region of cells in the IR group with condensed chromatin and fragmented DNA (Fig. 1C). The IR + Mel group exhibited a decrease in TUNEL positivity compared with the IR group (Fig. 1C). Upon quantification of viable cells in the hippocampi, the IR and IR + Mel groups exhibited a decrease in the mean number of surviving neurons, as compared with the control group. However, the number of surviving neurons were significantly increased in the IR + Mel group, as compared with the IR group $(\mathrm{P}<0.01$; Fig. 1D). These results suggest that Mel administration attenuates irradiation-induced damage.

Level of caspase-3 activity and mean ROS accumulation. Caspase-3 activity levels were significantly increased in the IR group compared with the control group ( $\mathrm{P}<0.01$; Fig. 2A). Treatment with Mel significantly attenuated caspase-3 activity levels in the IR + Mel group compared with the IR group $(\mathrm{P}<0.05$; Fig. 2A). As detected by a DCF probe, the mean ROS accumulation was significantly increased in the hippocampi of rats in the IR group compared with the control group ( $\mathrm{P}<0.01$; Fig. 2B). Furthermore, Mel administration significantly reduced ROS accumulation, and thus attenuated irradiation-induced damage, in the IR + Mel group compared with the IR group ( $\mathrm{P}<0.01$; Fig. 2B).

Mel administration attenuates caspase-3 expression levels. The expression levels of caspase- 3 were detected by western blot and RT-qPCR analyses. In the IR group, caspase- 3 expression levels were significantly increased compared with the control group, at the protein $(\mathrm{P}<0.01$; Fig. $3 \mathrm{~A}$ and $\mathrm{B})$ and mRNA ( $\mathrm{P}<0.01$; Fig. 3C) levels. Caspase-3 protein expression levels were significantly decreased in the IR+ Mel group compared with the IR group $(\mathrm{P}<0.01$; Fig. $3 \mathrm{~A}$ and $\mathrm{B})$, as were caspase-3 mRNA expression levels $(\mathrm{P}<0.05$; Fig. 3C). These results suggested that treatment with Mel significantly attenuated the expression levels of caspase-3. 


\section{Discussion}

In the present study, a modified protocol was used to induce hippocampal neurodegeneration by irradiation in vivo in order to investigate the potential protective mechanism of Mel on the hippocampi via decreased caspase-3 expression and activity levels. Radiation damage to cells is caused by oxidative stress (32). The increased caspase-3 levels detected in the IR group of the present study demonstrated the role of oxidative mechanisms in irradiation-induced tissue injury. Free oxygen radicals are molecules released from macrophages and neutrophils, which are efficient in the early period of inflammation, that target DNA proteins and lipids (33). Appropriate antioxidation intervention, via the inhibition or reduction of free radicals, offers protection against radiation-induced damage. Mel is a highly efficient free radical scavenger and a general antioxidant that has previously been demonstrated to protect DNA, lipids and proteins $(32,34,35)$. Furthermore, antioxidant enzyme activities have been shown to exhibit circadian rhythms that correspond to Mel rhythmicity and the total antioxidant status (36). Mel has been reported to increase the activity of important antioxidant enzymes at the molecular level, including superoxide dismutase and glutathione peroxidase (37). The present study investigated the antioxidant and immunoenhancing actions of Mel via the inhibition of caspase-3 in vivo.

As previous studies have demonstrated, Mel acts as a stimulant under immunosuppressive conditions or as an anti-inflammatory compound during immune responses, including acute inflammation (38-41). Advantageously, Mel is ubiquitously distributed in all the cellular compartments, and is capable of quickly passing through all the biological membranes (42). The results of a previous study demonstrated that no significant changes were identified in the Mel levels among the IR, IR + Mel and control groups $24 \mathrm{~h}$ after treatment (43). These results may be due to the ability of Mel to stimulate antioxidative enzymes which may maintain their enzyme activity levels following the metabolic decomposition of Mel. Therefore, signal transduction and the expression levels of antioxidant enzymes following treatment with radiation and Mel should be investigated in future studies.

In conclusion, the results of the present study demonstrated that Mel may protect hippocampal neurons from apoptosis via the inhibition of caspase-3 following irradiation. A significant decrease in caspase-3 mRNA $(\mathrm{P}<0.05)$ and protein $(\mathrm{P}<0.01)$ expression levels was detected at $24 \mathrm{~h}$ after Mel treatment. Therefore, caspase-3 inhibition may have a neuroprotective and antioxidant role in the interventional treatment of Mel, and these results demonstrate the potential therapeutic effect of Mel against irradiation. Further studies are required in order to elucidate the underlying effects and mechanisms of Mel on irradiation-induced alterations in caspase-3.

\section{Acknowledgements}

The present study was supported by the National Science and Technology Pillar Program during the 12th Five-year Plan Period (grant no. 2012BA127B02), by the Special Foundation of the Ministry of Health (grant no. 201002009), National Natural Science Foundation of China (grant nos. 31170804, 31240052,
31200634 and 81560025), the Natural Science Foundation of Tianjin (grant nos. 13JCYBJC23500, 13JCQNJC11600, 11ZCGYSY02400, 12JCYBJC15300 and 12JCYBJC32900) and the PUMC Youth Fund and Fundamental Research Funds for the Central Universities (grant nos. 2012G01 and 2012J05).

\section{References}

1. Li T, Lu C, Xia Z, Xiao B and Luo Y: Inhibition of caspase-8 attenuates neuronal death induced by limbic seizures in a cytochrome $c$-dependent and Smac/DIABLO-independent way. Brain Res 1098: 204-211, 2006.

2. Mansour HH, Hafez HF, Fahmy NM and Hanafi N: Protective effect of N-acetylcysteine against radiation induced DNA damage and hepatic toxicity in rats. Biochem Pharmacol 75: 773-780, 2008.

3. Shirazi A, Mihandoost E, Ghobadi G, Mohseni M and Ghazi-Khansari M: Evaluation of radio-protective effect of melatonin on whole body irradiation induced liver tissue damage. Cell J 14: 292-297, 2013.

4. Ashkenazi A and Dixit VM: Death receptors: Signaling and modulation. Science 281: 1305-1308, 1998.

5. Budihardjo I, Oliver H, Lutter M, Luo X and Wang X: Biochemical pathways of caspase activation during apoptosis. Annu Rev Cell Dev Biol 15: 269-290, 1999.

6. Vaux DL and Korsmeyer SJ: Cell death in development. Cell 96: 245-254, 1999

7. Wu H, Che X, Zheng Q, Wu A, Pan K, Shao A, Wu Q, Zhang J and Hong Y: Caspases: A molecular switch node in the crosstalk between autophagy and apoptosis. Int J Biol Sci 10: 1072-1083, 2014.

8. Du C, Fang M, Li Y, Li L and Wang X: Smac, a mitochondrial protein that promotes cytochrome $c$-dependent caspase activation by eliminating IAP inhibition. Cell 102: 33-42, 2000.

9. Srinivasula SM, Hegde R, Saleh A, Datta P, Shiozaki E, Chai J, Lee RA, Robbins PD, Fernandes-Alnemri T, Shi Y and Alnemri ES: A conserved XIAP- interaction motif in caspase-9 and Smac/DIABLO regulates caspase activity and apoptosis. Nature 410: 112-116, 2001.

10. Boland K, Flanagan L and Prehn JH: Paracrine control of tissue regeneration and cell proliferation by Caspase-3. Cell Death Dis 4: e725, 2013.

11. Sahoo S, Meijles DN and Pagano PJ: NADPH oxidases: Key modulators in aging and age-related cardiovascular diseases? Clin Sci (Lond) 130: 317-335, 2016

12. Sochocka M, Koutsouraki ES, Gasiorowski K and Leszek J: Vascular oxidative stress and mitochondrial failure in the pathobiology of Alzheimer's disease: A new approach to therapy. CNS Neurol Disord Drug Targets 12: 870-881, 2013.

13. Singh VK, Ducey EJ, Brown DS and Whitnall MH: A review of radiation countermeasure work ongoing at the armed forces radiobiology research institute. Int J Radiat Biol 88: 296-310, 2012.

14. Weiss JF and Landauer MR: History and development of radiation-protective agents. Int J Radiat Biol 85: 539-573, 2009.

15. Dumont F, Le Roux A and Bischoff P: Radiation countermeasure agents: An update. Expert Opin Ther Pat 20: 73-101, 2010.

16. Suman S, Jain S and Chandna S: Recent patents in the field of radioprotector development: Opportunities and challenges. Recent Pat Biotechnol 7: 219-227, 2013.

17. Poeggeler B,Saarela S, Reiter RJ, Tan DX, ChenLD, Manchester LC and Barlow-Walden LR: Melatonin-a highly potent endogenous radical scavenger and electron donor: New aspects of the oxidation chemistry of this indole accessed in vitro. Ann NY Acad Sci 738: 419-420, 1994

18. Wang Z, Liu D, Zhan J, Xie K, Wang X, Xian X, Gu J, Chen W and Hao A: Melatonin improves short and long-term neurobehavioral deficits and attenuates hippocampal impairments after hypoxia in neonatal mice. Pharmacol Res 76: 84-97, 2013.

19. Zhang Y, Cook A, Kim J, Baranov SV, Jiang J, Smith K, Cormier K, Bennett E, Browser RP, Day AL, et al: Melatonin inhibits the caspase-1/cytochrome $c /$ caspase- 3 cell death pathway, inhibits MT1 receptor loss and delays disease progression in a mouse model of amyotrophic lateral sclerosis. Neurobiol Dis 55: 26-35, 2013.

20. Jang SS, Kim HG, Lee JS, Han JM, Park HJ, Huh GJ and Son CG: Melatonin reduces X-ray radiation-induced lung injury in mice by modulating oxidative stress and cytokine expression. Int J Radiat Biol 89: 97-105, 2013. 
21. El-MissiryMA,FayedTA,El-SawyMRandEl-SayedAA:Ameliorative effect of melatonin against gamma-irradiation-induced oxidative stress and tissue injury. Ecotoxicol Environ Saf 66: 278-286, 2007.

22. Koc M, Buyukokuroglu ME and Taysi S: The effect of melatonin on peripheral blood cells during total body irradiation in rats. Bio Pharm Bull 25: 656-657, 2002.

23. Kim JS, Lee HJ, Kim JC, Kang SS, Bae CS, Shin T, Jin JK, Kim SH, Wang $\mathrm{H}$ and Moon C: Transient impairment of hippocampus-dependent learning and memory in relatively low-dose of acute radiation syndrome is associated with inhibition of hippocampal neurogenesis. J Radiat Res 49: 517-526, 2008.

24. Yang M, Song MS, Kim SH, Kim JC, Kim JS, Shin T and Moon C: Cytotoxicity of gamma-ray in rat immature hippocampal neurons. J Vet Sci 12: 203-207, 2011.

25. Kim JS, Yang M, Kim SH, Shin T and Moon C: Neurobiological toxicity of radiation in hippocampal cells. Histol Histopathol 28: 301-310, 2013.

26. Fike JR, Rosi S and Limoli CL: Neural precursor cells and central nervous system radiation sensitivity. Semin Radiat Oncol 19 122-132, 2009.

27. Kesari KK, Kumar S and Behari J: Pathophysiology of microwave radiation: Effect on rat brain. Appl Biochem Biotechnol 166: 379-388, 2012.

28. Li J, Feng L, Xing Y, Wang Y, Du L, Xu C, Cao J, Wang Q, Fan S, Liu Q and Fan F: Radioprotective and antioxidant effect of resveratrol in hippocampus by activating Sirt1. Int J Mol Sci 15: 5928-5939, 2014

29. Sharma S, Haldar $C$ and Chaube SK: Effect of exogenous melatonin on X-ray induced cellular toxicity in lymphatic tissue of Indian tropical male squirrel, Funambulus pennanti. Int J Radiat Biol 84: 363-374, 2008.

30. Livak KJ and Schmittgen TD: Analysis of relative gene expression data using real-time quantitative PCR and the 2(-Delta Delta C(T)) Method. Methods 25: 402-408, 2001.

31. LeBel CP, Ali SF, McKee M and Bondy SC: Organometal-induced increases in oxygen reactive species: The potential of 2',7'-dichlorofluorescin diacetate as an index of neurotoxic damage. Toxicol Appl Pharmacol 104: 17-24, 1990.

32. Yavuz MN, Yavuz AA, Ulku C, Sener M, Yaris E, Kosucu P and Karslioglu I: Protective effect of melatonin against fractionated irradiation-induced epiphyseal injury in a weanling rat model. J Pineal Res 35: 288-294, 2003.
33. Aritas Y, Akcan A, Erdoğan AR, Akgün H, Saraymen R and Akyildiz H: Effects of melatonin and phospholipid on adhesion formation and correlation with vascular endothelial growth factor expression in rats. Ulus Travma Acil Cerrahi Derg 15: 416-422, 2009.

34. Weiss JF and Landauer MR: Radioprotection by antioxidants. Ann NY Acad Sci 899: 44-60, 2000.

35. Miller E, Walczak A, Majsterek I and Kẹdziora J: Melatonin reduces oxidative stress in the erythrocytes of multiple sclerosis patients with secondary progressive clinical course. J Neuroimmunol 257: 97-101, 2013.

36. Albarrán MT, López-Burillo S, Pablos MI, Reiter RJ and Agapito MT: Endogenous rhythms of melatonin, total antioxidant status and superoxide dismutase activity in several tissues of chick and their inhibition by light. J Pineal Res 30: 227-233, 2001.

37. Majsterek I, Gloc E, Blasiak J and Reiter RJ: A comparison of the action of amifostine and melatonin on DNA-damaging effects and apoptosis induced by idarubicin in normal and cancer cells. J Pineal Res 38: 254-263, 2005.

38. Hardeland R, Madrid JA, Tan DX and Reiter RJ: Melatonin, the circadian multioscillator system and health: The need for detailed analyses of peripheral melatonin signaling. J Pineal Res 52: 139-166, 2012.

39. Carrillo-Vico A, Lardone PJ, Alvarez-Sánchez N, Rodríguez-Rodríguez A and Guerrero JM: Melatonin: Buffering the immune system. Int J Mol Sci 14: 8638-8683, 2013.

40. Espino J, Pariente JA and Rodríguez AB: Oxidative stress and immunosenescence: Therapeutic effects of melatonin. Oxid Med Cell Longev 2012: 670294, 2012.

41. Mate I, Madrid JA and De la Fuente M: Chronobiology of the neuroimmunoendocrine system and aging. Curr Pharm Des 20: 4642-4655, 2014.

42. Sainz RM, Mayo JC, Rodriguez C, Tan DX, Lopez-Burillo S and Reiter RJ: Melatonin and cell death: Differential actions on apoptosis in normal and cancer cells. Cell Mol Life Sci 60: 1407-1426, 2003.

43. Vijayalaxmi, Meltz ML, Reiter RJ, Herman TS and Kumar KS: Melatonin and protection from whole-body irradiation: Survival studies in mice. Mutat Res 425: 21-27, 1999. 